\title{
Consumers as Community Support Providers: Issues Created by Role Innovation
}

\author{
C.T. Mowbray, Ph.D. \\ D.P. Moxley, Ph.D. \\ S. Thrasher, D.S.W. \\ D. Bybee, Ph.D. \\ N. McCrohan, M.A. \\ S. Harris, M.A. \\ G. Clover, M.S.W.
}

\begin{abstract}
Using data from a CSP-funded research demonstration project designed to expand vocational services offered by case management teams serving people with serious mental illness, this paper examines the issues created by employing consumers as peer support specialists for the project. Roles and benefits of these positions are analyzed. Challenges experienced by specialists created by serving peers, the structure of the position, the mental health system and the community, and personal issues are analyzed using data from focus groups and the project's management information system. Implications for consumer role definition, supports for role effectiveness, and the structuring of these types of positions are discussed.
\end{abstract}

C.T. Mowbray is Associate Professor, School of Social Work, the University of Michigan, Ann Arbor, Michigan. D.P. Moxley is Associate Professor, School of Social Work, Wayne State University, Detroit, Michigan. S. Thrasher is Assistant Professor, School of Social Work, Wayne State University, Detroit, Michigan. D. Bybee is Adjunct faculty and N. McCrohan is a graduate student in the Department of Psychology, Michigan State University, East Lansing, Michigan. S. Harris is a consultant in East Lansing, Michigan. G. Clover is affiliated with Kent County Community Mental Health Board, Grand Rapids, Michigan.

Address correspondence to Carol T. Mowbray, Ph.D., The University of Michigan, School of Social Work, 1065 Frieze Bldg., Ann Arbor, MI 48109.1285. 


\section{INTRODUCTION}

Training and employment options are increasingly available to people with psychiatric disabilities. In fact, funding through the federal Community Support Program (CSP) and other state and local sources has established a number of programs where individuals work in mental health systems in which they are or have been service recipients. A recent survey of nearly 400 agencies which offer supported housing to persons with severe mental illness reported that $38 \%$ employed mental health consumers as paid staff (Besio \& Mahler, 1993). Reports on efforts to employ consumers are now emerging in the literature (Mowbray, Chamberlain, Jennings \& Reid, 1988; Mowbray, Wellwood \& Chamberlain, 1988; Howie the Harp, 1990; Cook, Jonikas \& Solomon, 1991; Stoneking, Greenfield, Sundby \& Boltz, 1991; Simon, 1992; Lysaker, Bell, Milstein, Bryson, Shestopal \& Goulet, 1993; Manos, 1993). Consumer jobs have included peer job coaches, case manager extenders, staff for drop-in centers, outreach workers, housing assistants, and many more. Besio and Mahler (1993) report salaries ranging from $\$ 3.35$ to $\$ 11.00$ an hour.

The involvement of consumers as mental health service providers has been galvanized by a growing consumer movement, expansion of disability rights, and the legitimization of self-help and peer support. Reasons cited for consumer employment are multiple. First, consistent with a rehabilitation philosophy, productive and important work is made available and accessible to consumers (NIDRR, 1992). Meaningful work contributes to increased self-esteem, acquisition of specific work skills, and perhaps career choices. Second, inclusion of consumers as mental health workers can increase the sensitivity of programs and services about recipients. That is, consumer employees are capable of better understanding clients' problems and their solutions, are better able to develop trust and rapport with clients, and better promote empowerment (Segal, Silverman \& Temkin, 1993). Third, they can serve as effective role models for clients, and fourth, the inclusion of consumers is an expression of affirmative action and consistent with contemporary civil and disability rights policies.

While reports are few, it appears that considerable complexity and resource requirements may be involved in establishing consumer employee positions. Additionally, these employees face stressors which may limit this vocational option. For example, confidentiality or having had a prior relationship with an assigned peer have been cited as problems (Besio \& Mahler, 1993) as well as clients who "test" the 
authority of their consumer-worker (Cook et al., 1991). According to some reports, consumer workers may suffer resentment or distrust from non-consumer staff fearing job displacement (Besio \& Mahler, 1993; Manos, 1993), experience problems with role definitions and boundary issues (Stoneking et al., 1991; NIDRR, 1992; Simon, 1992), or have difficulties relating personally to other workers (Lysaker et al., 1993); although other literature has endorsed consumer employees as being more able to establish friendships with assigned clients (Engstrom, Brooks, Jonikas, Cook \& Witheridge, 1991).

Most published reports, however, provide only brief descriptions of their programs. Little information is available on the specific challenges which consumer employees face or how these have been resolved. In contrast, this report provides operational details on consumer-employment and discusses its challenges. The program to be described successfully employed consumers as peer support specialists within a CSP-funded research demonstration project designed to increase vocational opportunities to clients already receiving case management services (Mowbray, Rusilowski-Clover, Arnold, Allen et al., 1994). Using data from the program's management information system, and from two focus group sessions held in the developing and fully operational stages of the project, we describe how consumer employees were involved in the rehabilitation team, the vocational services these workers provided, and the benefits and challenges they experienced personally, in interaction with the people to whom they provided support, and in the context of the mental health system.

\section{BACKGROUND}

\section{Description of Project and Participants}

Project WINS (Work Incentives and Needs Study) was funded as a three-year research demonstration project by the Center for Mental Health Services, SAMHSA. Its purpose was tt add resources to expand the vocational focus of seven existing case management teams (serving about 800 clients) in a large, suburban, community mental health service system located in Kent County, Michigan (See Mowbray et al., 1994). The principles guiding WINS' operations emphasized the Choose-Get-Keep mode of vocational rehabilitation (Farkas \& Anthony, 1989) self-determination (Moxley \& Freddolino, 1990), and zero exclusion, with eligibility based primarily on clients' self-selection rather than staff determination of work potential (Farkas \& Anthony, 1989). 
Demographically, clients eligible for WINS services were in their mid-thirties (median $=36$ ); the majority were white $(80 \%)$ and over half were male (60.4\%). Most graduated from high school, although educational levels ranged widely (four to 18). Functionally, clients fit a definition of chronic mental illness widely accepted by rehabilitation and community support models of service delivery (Lawn \& Myerson, 1993). The predominant diagnosis was schizophrenia (41.6\%), followed by affective disorders (20.6\%). Most participants were experiencing moderate symptoms and functioning with some difficulty and nearly all were currently taking psychotropic medication (See Mowbray, Bybee, Harris, \& McCrohan (1995) for more details.). During the 18 months between February, 1992 and July, 1993, 263 individuals were enrolled in Project WINS.

The major component of WINS' operations were the services of five Vocational Specialists (VS's) - professionals assigned to the case management teams, who provided direct services to a rotating caseload of about 25 clients each, along with case consultation and overall information about vocational issues to case managers from the teams.

\section{Consumer Employees}

Clients could also elect to receive services from Peer Support Specialists (PSS's)-consumers who worked with a VS as a case manager extender. The PSS provided services to assigned individuals as negotiated in their WINS intervention plan; for instance, helping clients to prepare resumes, set up bank accounts, acquire clothing for interviews or work, or learn the bus system. PSS's were also responsible for facilitating Job Support Groups, producing a newsletter, and carrying out other informational activities for WINS.

PSS's were referred by their case managers, interviewed by the WINS director, and hired if there was a mutual decision that the position was appropriate for them. Three weeks' preservice training was provided before they started working with clients, focusing on conflict resolution, stress management, self-help principles, and operational issues. PSS's salaries ranged from five to seven dollars an hour, with employment varying from a few hours up to 30 per week, depending on availability, desire, and financial need. The compensation was based on face-to-face or telephone contacts with peers, or scheduled activities in the agency.

Project WINS originally intended to hire consumers as job coaches but once implementation began it was obvious that many participants had the skills to do their jobs. Rather, assistance and support were 
needed around getting and keeping jobs. The PSS role, therefore, evolved to encompass a wide range of community support activities.

\section{Characteristics of PSS's}

Over 24 months of project operations, 19 PSS's completed training and worked on the project: 11 males and 8 females, average age of 34.8 years. Two were African-American; one was Hispanic; the remainder were nonminorities. The average educational level of PSS's was 13.5 years (range 11-16). PSS's experiences with the mental health system varied: of those for which data were available $(n=9)$, all had one or more psychiatric hospitalizations; most had more than one (range 1-7). Length of these hospitalizations varied from two to 314 days, with a median of 11 days. (Longer hospitalizations had occurred 8 or more years previously.) Hospitalization dates ranged from 1982 up to the present (including some which occurred while being PSS's). On average, PSS's had been community mental health clients for 6.7 years (range 1-13).

\section{METHOD}

\section{Management Information System Data}

Service Activity Logs were submitted weekly by all direct service staff (vocational specialists and peer support specialists) to the agency's Information System from $2 / 92$ through 7/93. Logs provided daily records of the amount of time each staff person spent in specific activities with identified clients, as well as work time not associated with individual clients (meetings, supervision, training, paperwork, etc.).

\section{Focus Group Data}

All PSS's who had completed training since the beginning of the project were invited to attend a focus group session after 15 months of operations. A year later, when the project was fully operational, all currently employed PSS's were invited to a second focus group. All attenders were compensated for their time. Of the eleven individuals attending the first session, about half were then employed as PSS's; the others were involved with employment, school, and/or training. All six attending the second focus group were working as PSS's. For both meetings, focus group participants were given copies of the focus group questions in advance and told that the purpose of the session was to get a better idea of how the project was operating, so that improvements could be made. Individuals were assured of the confidentiality of their individual responses and that they could review the focus group summary. All participants agreed to taping the session. The facilitator and one other project staff attending took notes during the session. The tapes were reviewed by consultants external to the project and consensus was reached on the results summarized below. 


\section{RESULTS}

\section{Quantitative Summary of PSS Work}

Ten PSS's were employed over the 18-month period for which records were available. Of the 263 persons served by WINS during this time period, 117 or $44 \%$ received some direct service from a PSS. Those not electing to work with a PSS cited the adequacy of their existing support systems or felt unclear about how a peer could provide vocational assistance. Ninety-five of the 117 who had contact with a PSS received substantial service (at least 1 hour of direct contact). Table 1 summarizes data from the management information system on PSS employment and the services provided to clients. Typically, between 5 and 6

TABLE 1

PSS Employment Information and Services Provided

\begin{tabular}{|c|c|c|c|c|}
\hline & \multicolumn{3}{|c|}{ Range } & \multirow{2}{*}{$\begin{array}{l}\text { Total } \\
\text { Possible }\end{array}$} \\
\hline & Median & Min. & $\operatorname{Max}$ & \\
\hline \multicolumn{5}{|l|}{ PSS Employment Data } \\
\hline \# PSS's working/month & 5.5 & 4 & 7 & 10 \\
\hline \# mos. employed & 13.5 & 3 & 18 & 18 \\
\hline \#rs. worked/month & 29.4 & 11.7 & 44.0 & 171.3 \\
\hline$\%$ hrs. direct client contact & $28 \%$ & $11 \%$ & $46 \%$ & - \\
\hline $\begin{array}{l}\% \text { hrs. indirect service: job dev., } \\
\text { marketing, activs. on behalf of clients }\end{array}$ & $8 \%$ & $5 \%$ & $25 \%$ & - \\
\hline$\%$ hrs.-peer support groups & $25 \%$ & $15 \%$ & $53 \%$ & - \\
\hline$\%$ hrs. - meetings & $13 \%$ & $2 \%$ & $27 \%$ & - \\
\hline$\%$ hrs. - paperwork & $9 \%$ & $1 \%$ & $9 \%$ & - \\
\hline \# supervisory hrs./mo. & 11.2 & 4.2 & 28.9 & \\
\hline \multicolumn{5}{|l|}{ PSS Service Provided } \\
\hline $\begin{array}{l}\text { Total \# clients assigned to all PSS's (per } \\
\text { mo.) }\end{array}$ & 29.5 & 22 & 40 & 117 \\
\hline Average caseload (per PSS/mo.) & 4.7 & 1 & 11 & - \\
\hline $\begin{array}{l}\text { Total \# clients served per PSS (over } 18 \\
\text { mos.) }\end{array}$ & 15 & 5 & 33 & - \\
\hline $\begin{array}{l}\text { Total \# served per PSS, w/subst. cont. } \\
\text { (over } 18 \text { mos.) }^{\text {a }}\end{array}$ & 12.5 & 2 & 20 & - \\
\hline Duration of service contact (in mos.) & 3 & 1 & 18 & 18 \\
\hline $\begin{array}{l}\text { Intensity of service contact }{ }^{\mathrm{a}} \text { (per } \\
\text { client/mo.), in hrs. }\end{array}$ & 2 & .35 & 10 & - \\
\hline
\end{tabular}

for cases receiving substantial PSS service (i.e., at least one hour of direct contact) 
PSS's were employed per month, and they worked about 7 hours per week, for somewhat more than a year's duration. Five of the PSS's worked continuously, with no breaks in direct service of a month or more; the other five each had an intervening break lasting one to five months (median = 3.5). About half the PSS time was equally divided between direct client contact and running support groups. The activities of indirect services, paperwork and meetings each used about another 10-15\% of time. The percent of time PSS's typically spent on direct service was comparable to that for the vocational specialists (VS's). Supervision of PSS's was provided by the VS's and over the total project period required each to spend about 10 hours per month per PSS. However, supervision hours declined steadily over time (21.3 hours per month average during the first quarter of the 18-month period to 7.9 during the last quarter). This decline was not related to fluctuation in caseload size or PSS workload and appeared to be attributable to the passage of time, improvements in VS staff experience, and perceived or actual PSS expertise. (Changes in reporting practices may also be reflected.) Efficiency in supervision was also noted over time, with the ratio of direct client contact hours provided by PSS's per hour of supervision increasing steadily (from 2.2 in the first month to 12.4 in the final month, median=6.1).

The PSS staff worked with about 30 clients per month. The usual caseload carried by a PSS was about 5 clients. Over the course of their employment, a PSS had typically served 15 clients. Generally, PSS's who worked the most months served the greatest number (Pearson's $r=.68$ ). The usual service duration was 3 months per client and intensity was 2 hours per month of direct contact. However, these median values belie the extreme variability in the data. For example, some clients received up to 10 hours per month of direct contact. Individual PSS's monthly caseloads also varied widely-serving as few as one or two clients in some months and more than 10 in others. Similarly, variation in the number of hours PSS's worked per month was also high (10 or fewer hours in some months and more than 40 in others; median interquartile range of 20 hours).

\section{Focus Group Results}

The focus group sessions provided an elaboration of the PSS role as well as an identification of challenges presented to PSS's personally, by their role, by the CMH system, and by the structure of the PSS position itself. 
Roles and Benefits of Peer Support Specialists. PSS's described the complexity of their job role: it was like being a friend, but different because you were expected to help, to educate, and to provide leadership. Several felt that their most important function was to encourage clients (reinforcing past job accomplishments, developing interests, suggesting alternatives, and/or facilitating self-determination). Others mentioned specific activities like counseling on personal hygiene, training on how to use public transportation, helping someone find a job, jobsite assessment, or conducting group sessions (e.g., resume writing). Some mentioned the importance of trying to change attitudes about the vocational abilities of individuals who are mentally ill-those of employers and mental health professionals. Through their formal involvement in the mental health system, PSS's facilitated communication of a consumer perspective to improve services. They believed the PSS role underscored the mental health system's commitment to consumerism and its willingness to take consumers seriously.

There was general agreement on the value of relating to the assigned peer (having a common bond, building trust, "show people we love them") and of role modeling. At the second focus group, PSS's especially stressed the importance of having a structure with clients, but remaining flexible within it; such as, setting regular appointments and keeping one's commitment, but accommodating disruptions and discussing their circumstances.

The PSS's were highly positive about the benefits of their service: "Mentally ill people feel alienated from the world. You need someone to talk to and be around with who is like you." They described several positive outcomes in clients' job successes-cashier, cook, factory worker, hi-lo truck driver. PSS's were also sensitive to important psychosocial benefits which can accompany successful employment, such as autonomy, confidence, and other associated rehabilitation outcomes, i.e., getting out of a negative living situation.

Beside the benefits that recipients accrued from working with consumer employees, the PSS's also focused on the benefits they personally experienced from their roles. "T've seen my peers grow and I've grown with them. It's been a sharing thing and really rewarding to me." Growth came about from being sensitive to the emotional states of clients, fulfilling commitments, acknowledging mistakes, and learning from them. They also described developing specific skills and talents (e.g., "I found I could talk in front of groups-not a trait I thought I had"), improving communication abilities ("T found I'm getting more information by getting feelings out than letting them stay inside me"), 
and increasing confidence ("I felt it was an honor [being employed as a PSS]-mental health workers treated me as an equal"). A lot of these changes occurred because of the unspoken respect they sensed from other clients ("People respect your wisdom-real rewarding") as well as the positive comments they received from professionals. Finally, many PSS's expressed the feelings of pride they experienced when one of their peers reached a vocational goal ("She was able to develop her own independency thinking").

Challenges Involved in Serving Peers. Dealing with the behaviors of the clients they worked with were the most frequently mentioned challenges, especially at the first focus group session. Particularly problematic were clients who were seen as "unmotivated": showing up late or not at all for appointments; saying they changed their minds about working, etc. Such behavior was frustrating because it caused the PSS's to waste their own time and lose compensation and also because of the implication that the PSS contact (and possibly the PSS him/ herself) was not valued by the client. Similar complaints are often voiced by non-consumer mental health staff. Another set of problems revolved around clients' continued use of drugs and alcohol. PSS's reported feeling personally let down in several cases when clients failed to maintain sobriety and consequently lost employment and all the gains that they had worked on so hard together ("It was my biggest disappointment").

At the second focus group, PSS's mentioned problematic client behaviors less frequently. Rather, they emphasized the importance of persisting with clients at all stages. They also talked more about needing to know their own limits and recognize when they were unable to perform their roles with clients. The ability to take a respite and say no at times was a foundation which allowed them to return. Responding in this way to their emotions appeared to safeguard the gains they had made.

Challenges Presented by the Mental Health System and the Community. The larger mental health system also created challenges for the PSS's. One PSS related how he felt let down when a client he had workee with successfully was discharged from WINS and then fired seven days later. He felt that the client did not want to leave WINS and that the case management agency had failed to follow through with the appropriate level of support and assistance. 
PSS's felt that the larger community erected barriers that frustrated the attainment of vocational goals for people with serious mental illness. One PSS described his own experiences in having "wobbly legs" during a job interview, due to medication side effects, and not getting the job. He went on to describe WINS clients: "When I work with them, I can see mental illness in their faces... Our society doesn't want people like that. I feel helpless, like I can't do anything about this."

The PSS's awareness of contemporary policy was revealed in a discussion of the Americans with Disabilities Act. They contemplated whether vocational opportunities would open up as the rights articulated by this legislation began to be understood more broadly within the community. This observation of helplessness underscores the necessity of placing affirmative employment of consumers in the mental health system within a broader societal context of continued stigma, discrimination, rejection, and rights violations.

Personal Challenges for the PSS. Some of the challenges personally experienced by the PSS's were identified as positive; for example, to develop flexibility, to "become vulnerable to strangers", and to acquire new knowledge (e.g., riding the bus). The issue of whether the PSS was a friend or a service provider, however, created dilemmas and stresses (whether or not to give out a home phone number, how to handle a client asking for a date, personal attraction to an assigned peer, or wanting to do "fun" things together). Several PSS's articulated knowing that their role was other than that of a friend but they still had difficulty translating this in particular situations.

Several of the PSS's expressed a real personal investment in their work. The greatest disappointments they described involved clients who "failed them." One PSS implied that he left the position because he worried about not being able to help people enough: "What worried me the most . . they're just like me ... we deal with the same things. So what can I contribute to them without upsetting them?" Another expressed mixed feelings when an assigned client decompensated on the job and he could not help him; he stated his relief, intermingled with sadness, in realizing that the client's situation was beyond the realm of a PSS. When assigned to work with a peer who was chemically dependent, another PSS experienced conflict due to personal involvement with a substance abuse 12-step program: "They wanted me to keep him sober ... but the 12 step program teaches that you're powerless over somebody else's drinking." 
Boundary issues were mentioned by at least half of the PSS's. Two men made specific statements about being able to maintain separateness ("I knew I was not supposed to take on their problems;" "I knew this was a job"). Many others indicated that it was difficult to "know when to draw the line" on the friendship and support components of their jobs. They described their own emotional involvement in the client's outcome. One PSS saw himself as "guardian of the client's selfdetermination rights." Another related that when he first met the peer he was assigned to he felt, "We could communicate... We could help each other out. We help them, they help us." Then the client repeatedly failed to show up for appointments, causing a major letdown for the PSS, who was never able to build a relationship with this individual. Another PSS described her feelings toward her assigned peer when he showed up drunk: "I could care less if you're going to act like that. I don't owe you anything." This same woman was able to summarize quite articulately the difficulties she faced working as a PSS:

I need to be detached from people who have problems like mine because it tends to trigger my anger, which triggers my delusional thinking. I have difficulties keeping boundaries with these people because they're so much like me.

While most of the PSS's did not have as much understanding about these issues, the mention of similarities with assigned peers was much more common than mention of differences: "People I deal with are people like myself . . . I have mental problems."

Boundary problems could be exacerbated when a PSS was assigned to work with an individual they already knew, which was difficult to avoid in this suburban community. A young male PSS was assigned a client he had known when the PSS was a child. The client's acceptance of the PSS's advice was difficult and the PSS felt a loss of credibility: "I felt bad because I wasn't there emotionally when needed. I didn't know what to say." Several other PSS's experienced conflicts when they worked with someone they wanted to have as a friend, not knowing how or whether to do this.

One PSS said her challenge was staying focused so that her thoughts did not interfere in an adverse manner with her role with clients. Techniques she employed to achieve this included learning to listen, making eye contact, and writing things down after each session. Other problems mentioned were keeping up with the job requirements despite continual car problems and overcoming fears about traveling in certain parts of the city to make visits to clients. 
Challenges Regarding the Structure of the PSS Position. Low pay rates posed difficulties for several PSS's who wanted jobs with sufficient compensation and fringe benefits to emancipate themselves from disability income. Fluctuating pay because of the hourly (versus salaried) nature of the job created problems, especially when it was due to factors beyond the control of the PSS; for example, clients who missed appointments, or couldn't be contacted; or when one client was discharged and his or her replacement did not want a PSS.

The fact that client contacts were usually scheduled on-site and at the client's convenience meant that PSS's often had a lot of "down-time" waiting for an appointment. For some PSS's, there seemed to be continual discussion about what constituted "compensable" time: e.g., phone contacts with a discharged client; those of an apparent social nature; transportation time to appointments; reading or thinking time. These disagreements contributed to feelings of inequity, an absence of teaming, and exploitation for the PSS's involved.

Other complaints voiced by PSS's concerned lack of information needed to do their jobs: not knowing what was in the peer's service records, or more training and/or guidance in service techniques. There was also some lack of clarity concerning how much autonomy the PSS position had. Project procedures specified that critical problems should be directed to the VS. However, if the VS was unavailable, some PSS's felt they could not then go to the peer's case management team and alert them. Others felt that in having to go through the VS, they were being treated as second class citizens, not members of the treatment team: "We're like gophers; we can't communicate directly." PSS's also expressed a desire for equity with the VS's in contact and communication with employers of the consumers they served. The problems concerning autonomy and knowledge seemed less central in the second compared to the first focus group.

\section{DISCUSSION}

State of the art psychosocial rehabilitation and community support practice stresses the importance of achieving consumer involvement in all phases of service delivery (Flexer \& Solomon, 1993). In designing WINS as an innovative service demonstration, to integrate vocational activities into case management teams, the inclusion of a consumer employment component was consistent with a rehabilitation value base and the programmatic principles of community support. But as indicated by the PSS 
focus group results, and verified by the perspectives of administrators, implementation of the PSS component was much more complex and difficult than had been imagined. Much of the contemporary literature in this area does not speak to the many issues which can emerge to compromise the involvement of consumers as mental health workers in community support projects. Our discussion attempts a fuller exposition of the implications of consumer service employment for psychosocial rehabilitation programs. We focus on PSS role activities, the provision of necessary supports for consumer workers, and the structure of this work to maximize benefits to consumer employees, to the mental health system employing them, and to the ultimate recipients.

\section{PSS Role Activities}

Project WINS represented an effort by one community support program to expand role opportunities and definitions for people with serious mental illness. The experiences of the project demonstrated the feasibility and value of involving people with serious mental illness as service providers although this role innovation does not come without challenges and perhaps problems. Focus group findings reflect the distinctive contribution that consumers can make as community support workers. The PSS workers appear to define their roles in flexible ways and demonstrate a willingness to undertake critical instrumental support activities such as assistance with hygiene, transportation, skill development, and job search skill development. Fully credentialed mental health professionals may not be willing or able to offer these services as part of community support and/or clinical role definitions. However, such roles appear to be essential to the effective execution of community support philosophy and values.

The nature of the relationship that PSS's offered to consumers was also identified as a distinctive contribution. Certainly fully credentialed mental health professionals offer consumers supportive and caring relationships. But the identification factor in which one peer can offer another a sympathetic understanding of mental illness-something which is often defined out of professional-consumer relationships-may add a special form of support and perhaps intimacy that can lower the social distance between provider and recipient. This identification with the "situation of the other" and a firm understanding of the daily challenges created by mental illness can promote the relevance of supports, contribute to advocacy with employers, and underscore the necessity of interpreting mental illness to the larger society. 
The information collected suggest that the role of PSS was not simplistic and "nonprofessional" but appeared to require considerable role sophistication involving the necessity to muster and sustain a positive attitudinal set, exercise important community support skills, and maintain daily motivation. Tasks such as job searching, job development, group facilitation, employer education, and trouble-shooting in the community all gave a professional tone to the role of PSS's.

\section{Role Definition Problems}

Yet, all three of these distinctive attributes-instrumental support, relationship building, and role sophistication-appeared to establish a context for role strain and role conflict for consumers who took on positions as community support workers. Relevant to the former, the requirements of these positions did not appear to be recognized and rewarded in terms of pay, discretion in the use of time, or decisionmaking autonomy. Material identified in the focus groups certainly suggested the possibility of a "degraded self-image" in which some consumers felt like "second class workers." In organizational cultures that prize education and practice credentials and which are "status conscious" (i.e., those operating in many mental health systems), the establishment of statuses which are not valorized for their contribution can readily lead to the devaluation of the incumbents of these positions.

Despite the arguments made in the literature for the creation of consumer employment positions in mental health settings and despite the positive benefits that result, as reported in this paper, there was considerable ambiguity in the status of PSS. As consumers become more successful in these roles and increasingly make important contributions to the support of their peers, growing expectations about the quality of work life and more assertiveness evolving from feelings of empowerment may result in heightened dissatisfaction with their employment status. Then, job dissatisfaction, lowered productivity, and turnover-problems professionals may mistakenly attribute to the poor performance of people with mental illness-may be more indicative of the ambiguity which accompanies the fusion of two different roles into one status. The resulting role strain could create a significant source of stress.

Thus, the risk of role strain among consumer employees must be addressed proactively by mental health systems seeking to offer this opportunity. Role conflict must also be taken into consideration, as evidenced by PSS reports that the relationships between peers became problematic 
when one remained a "client" and the other became a "worker." The special sympathy consumer workers have toward their peers may be eroded when these individuals do not perform as "good clients." The impact of this may be amplified when the income of the consumer worker is tied directly to the client's performance and behavior. Resentment toward a peer may increase when this person does not make appointments, fails to show for contacts, or changes his or her mind about seeking employment. Psychologically, consumer workers may struggle with ambivalences created by these feelings and perhaps react negatively towards their peers, showing anger, blame, and rejection.

Of course, there are other boundary issues as well. Several PSS's identified the possibility of relating to their clients as friends or even significant others. How and when to draw the line may introduce more ambiguity into the role definition of the consumer worker. For some PSS's, the answer was straight forward: reduce the role conflict by giving up the status of mental health worker. But what are the implications for those individuals who continue to struggle with this challenge? Do they decrease their special identification with the other and the sympathy they offer? To resolve this conflict consumer workers may have to relinquish those very characteristics that enable them to make a distinctive contribution to community support.

The challenges to role innovation may be expressed as questions consumer workers pose to themselves but nonetheless must be addressed by their employers: Am I consumer or worker? What is my status? Am I valued? Will I be treated more professionally as the scope and substance of my expertise expands? How do I frame my role to my peer-clients? Do the rewards exceed the problems I experience?

\section{Suggestions for Supports and Structures to Increase Role Effectiveness}

There are no easy solutions to the issues and problems raised by those questions. Yet, the questions themselves require community support systems that plan to engage in consumer role innovation to contemplate ways to support the effectiveness of consumer employees. We consider these supports in the context of a progressive human resource system which requires: (a) the establishment of a mission and culture conducive to consumer role innovation; (b) the availability of mentoring and consistent supervision; and (c) the establishment of opportunities for education and advancement. 
The Mission and Culture of the Community Support System. Changing the roles of consumers by creating opportunities for work as mental health service providers may be a change in the expressed purpose and mission of the community support program. Rather than being a system in which professionals are dominant, the community support system is elaborating opportunities for other stakeholders, in particular, active or former consumers. This change needs to be interpreted to all those involved in the effort as well as to the general mental health system. The rationale guiding this change and the nature of the opportunities available to consumers must be communicated consistently especially internally. Merely to frame this effort as a means to extend services or to increase the sensitivity of service provision probably falls short of a recognition of its profound impact on the self-concepts and identities of consumers accepting these new roles. Perhaps it is critical to include within this mission the goal of opening up affirmative employment for people with serious mental illness to assist them to develop viable career opportunities within community support systems. The establishment of this aspect of the mission clearly communicates to everyone-including professionals who may dismiss consumer workers as paraprofessionals or as aides - that the community support system is seeking seriously to expand role options and work opportunities.

But specifying a new mission will not address fully the role challenges posed earlier. It is, however, a start to restructure the cultures of many mental health systems which are inordinately dependent on hierarchy, professional credentials, and accreditation requirements. Addressing some of the expected conflict between professionals and newly empowered consumer workers with the aim of modifying organizational culture can be approached through several strategies. First, an educational campaign can be launched to interpret role innovation and the new policies the community support system will follow in its implementation. Second, the relationships between professionals and consumer workers can be the focus of team meetings. Third, performance expectations for supporting the effectiveness and development of consumer employees and the achievement of the new mission can be communicated directly to front-line supervisory staff. These are the positions most likely to have administrative interaction with consumer employees. Assuring that these individuals support these new workers is probably essential to the overall effectiveness of role innovation.

The Provision of Mentoring and the Role of Supervision. The necessity of consistent mentoring and supervision of consumer employees 
cannot be over-emphasized. As previously discussed, many consumer employees will enter work situations characterized by considerable role ambiguity. Although many new workers experience ambiguity when they initially enter programs, this may be particularly difficult for consumer workers because of the relative novelty of their roles. The availability of mentoring will assure that consumer workers have another senior individual (perhaps a senior consumer worker or a professional staff member) who they can turn to for support, trouble-shooting, wisdom, or extra problem-solving assistance. The role of the mentor is to assist the consumer worker to master the demands of organizational life within the community support system.

Mentoring does not replace supervision which is designed to assist consumer workers to master the particular requirements of the job, effectively execute relevant policies and procedures, and manage their time and productivity. Consistent supervision within teams as well as supervision designed for consumer workers as a group should increase the probability that workers will have confidants available to them when they need assistance in addressing role and organizational demands. In WINS, we found that initially in the Project PSS supervisory demands were high: requiring approximately one-third of the total direct time the PSS's spent with their assigned clients. However, over the duration of the project, supervisory contacts were markedly reduced, to less than $10 \%$ of total direct client contact time. This increased supervisory efficiency undoubtedly reflects increased proficiency on the part of the PSS's in doing their jobs and may also reflect improvements in the VS's ability to provide them with the direction they need.

Opportunities for Education and Advancement. Complementing mentoring and supervision is the availability of an in-service education program offering content specifically designed to augment the skills, competencies, attitudes, and motivation of consumer workers. Content that targets problems and issues experienced directly by consumer workers not only recognizes symbolically the demands placed on such workers but also pragmatically addresses these demands through skill development opportunities. Mentoring, supervision, and in-service education will enable the organization to support the continuous definition of the roles of consumer workers and assist these workers in forging a positive and productive self-identity over time.

Data from Project WINS indicates that consumer workers can fill gaps by offering pragmatic supports to people with serious mental 
illness, supports that can go unaddressed and lead to serious unwanted outcomes. There is no reason why consumer workers cannot be recognized for their performance as the nature, sophistication, and productivity of their contribution to community support grows over time. Compensation systems which are based on educational credentials and which separate professionals and consumers may overlook the pragmatic nature of community support work and result in the devaluation of consumers' contributions.

While recognizing appropriate limitations on their assigned responsibilities and their status as workers in training, the compensation of consumer workers can occur in a number of ways including higher hourly wages, increased work weeks, benefits, and promotion. In the WINS program, our initial structuring of the PSS job utilized hourly rates to allow flexibility in work demands across clients and for the same client over time. But this structure had the unintended consequence of producing stress because the fluctuating client loads produced fluctuating income levels. It also suggested a fundamental lack of trust through activities to oversee and review whether the time charged to WINS was really "appropriate." Although PSS's may not have wanted to be professionals, they sensed they were not being treated professionally, as this comment suggest: "We don't get paid the time for thinking or processing that the others do." Compensation and the monitoring of work should reinforce the identity of consumer workers as employees who can make vital contributions, who develop over time, and who conduct themselves professionally.

Hiring consumers into specially identified positions, such as PSS may be inherently problematic. It could be argued that creating a programmatic culture in which there are dual tracks for professionals and consumer workers will only foment divisiveness, raising equity and justice issues. Can both types of staff be treated equally? Should the special perspective and experiences brought to the work situation by consumer workers be recognized and compensated at a level and in a form that respects their distinctive contributions? Failure to address a fair and progressive performance-based pay system that includes assessment, planning, travel, and case finding efforts may only suggest that consumer workers constitute a superfluous service, appended to meet an ostensible "commitment to consumer involvement." Community support systems engaging in consumer role innovation must address the question of "where do these opportunities lead the consumer worker?" by explicating whether positions are for training purposes or whether they have career potential. These two different approaches 
should be communicated in a program's statement of mission. If the program is committing itself to training, then the length of the training, expected competencies, linkage to other jobs and careers, and supports for transition in employment should be made explicit.

If consumer employment is an expression of affirmative action, sponsoring programs must give thought to career ladders for consumers who decide to remain as workers, so that the mental health system can become a source of upward mobility. The nature of the career ladder must be operationalized, the criteria for advancement identified, and the range of opportunities communicated. For example, can consumers who complete a probationary period successfully enter a salaried position, work at a permanent full or part-time position, obtain benefits, and advance to other levels or forms of employment within the system? Explication of this career ladder will mean that interested and motivated consumer workers can eventually leave that status completely and assume other roles within the community support system.

\section{CONCLUSIONS}

Project WINS demonstrated the efficacy and problems inherent in consumer role innovation. To conclude that consumer employment is either flawless or fraught only with problems is erroneous. A more balanced conclusion is called for based on the experience of Project WINS: Consumers as community support workers can make distinctive contributions to the support of their peers within the community and on the jobsite. These include the provision of pragmatic and meaningful instrumental supports, the formation of relationships that are different from those that occur between professionals and consumers, and the potential to enhance motivation of consumers and professionals alike by modeling successful roles. However, we must also point out that role innovation can create considerable ambiguity, role conflict, role strain, and personal stress for the incumbents of these positions.

The development and expansion of employment opportunities within community support systems is a natural extension of the philosophy, values, and goals of this programmatic model. Yet, consumer workers cannot be treated as mere instrumentalities indicative of some kind of nominalistic commitment to consumer involvement or merely reflective of a new strategy for organizing services. This role innovation must contribute to the development of consumers themselves-for those who assume the roles as workers and for those who receive services from 
their peers. Perhaps the next step in understanding consumer role innovation is to increase our knowledge of how such innovation makes an impact on these consumer-workers aside from its effects on the community support system. Suffice it to say at this juncture, that consumers who took on new roles as workers within Project WINS experienced significant developmental challenges which, for some, resulted in the redefinition of themselves, the discovery of new personal capacities, skills, and strengths, and the establishment of new employment and career vistas.

We must also conclude that role innovation does not occur by merely opening up new opportunities for consumers. Organizing and supporting this innovation effectively is of paramount importance and requires community support systems to rethink and perhaps redefine their mission and goals, programmatic cultures, human resource development systems, career development ladders, and performance compensation policies. The data obtained through Project WINS suggest that the stress for consumer workers may be too high without a reconfiguration of organizational supports for this type of innovation.

Certainly community support systems are seeking ways of becoming more client-driven in their mission, policies, and practices. Consumer role innovation which offers consumers new opportunities and positions as workers within such systems appears to be one means of further moving toward the ideal of client-driven service delivery. However, consumer employment within our systems must be seen from a dual perspective: as a strategy that can improve the accessibility, sensitivity, and competence of the systems themselves, and as a strategy that can significantly impact outcomes for consumer workers.

\section{REFERENCES}

Besio, S.W. and Mahler, J. (1993). Benefits and challenges of using consumer staff in supported housing services. Hospital and Community Psychiatry, 44, 490-491.

Cook, J.A., Jonikas, J.A., \& Solomon, M.L. (1991). Models of vocational rehabilitation for youth and adults with severe mental illness. American Rehabilitation, 17.

Engstrom, K., Brooks, E.B., Jonikas, J.A., Cook, J.A., Witheridge, T.F. (1991). Creating Community Linkages: A Guide to Assertive Outreach for Homeless Persons with Severe Mental Illness. Chicago, IL: Thresholds, Inc.

Farkas, M. and Anthony, W.A. (1989). Psychiatric Rehabilitation Programs: Putting Theory into Practice. Baltimore: Johns Hopkins University Press.

Flexer, R. \& Solomon, P. (1993). Psychiatric Rehabilitation in Practice (pp. 137-153). Boston: Andover Medical Publishers.

Gilbert, N. \& Specht, H. (1974). Dimensions of Social Welfare Policy. Englewood Cliffs, N.J.: Prentice-Hall. 
Howie the Harp (1990). Independent living with support services: The goal and future for mental health consumers. Psychosocial Rehabilitation Journal, 13, 85-89.

Lawn, B. \& Meyerson, A. (1993). A modern perspective on psychiatry in rehabilitation. In R.W. Flexer \& P.L. Solomon. Psychiatric Rehabilitation in Practice (pp. 31 43). Boston: Andover Medical Publishers.

Lysaker, P., Bell, M., Milstein, R., Bryson, G., Shestopal, A., Goulet, J.B. (1993). Work capacity in schizophrenia. Hospital and Community Psychiatry, 44, 278-280.

Manos, E. (1993). Speaking out. Psychosocial Rehabilitation Journal, 16, 117-120.

Mowbray, C.T., Bybee, D., Harris, S.N. \& McCrohan, N. (1995). Predictors of work status and future work orientation in people with a psychiatric disability. Psychiatric Rehabilitation Journal, 19, 17-28.

Mowbray, C.T., Chamberlain, P.J., Jennings, M. \& Reed, C. (1988). Consumer-Run Mental Health Services: Results from Five Demonstration Projects, Community Mental Health Journal, 24, $151-156$.

Mowbray, C.T., Rusilowski-Clover, G., Arnold, J., Allen, C., Harris, S., McCrohan, N., \& Greenfield, A. (1994). Project WINS: Integrating Vocational Services on Mental Health Case Management Teams. Community Mental Health Journal, 30(4), 347-362.

Mowbray, C.T., Wellwood, R. \& Chamberlain, P.J. (1988). Project Stay: A Consumer-Run Support Service. Psychosocial Rehabilitation Journal, 12, 33-42.

Moxley, D.P. \& Freddolino, P. (1990). A model of advocacy for promoting client self-determination in psychosocial rehabilitation. Psychosocial Rehabilitation Journal, 14(2), 69-82.

National Institute on Disability and Rehabilitation Research (NIDRR). (1992). Consensus Statement, 1(3). Washington, D.C.: Author.

Segal, S.P., Silverman, C., \& Temkin, T. (1993). Empowerment and self-help agency practice for people with mental disabilities. Social Work, 38, 705-712.

Simon, M. (1992). Making a difference: Ex-patients as staff. Resources. Newsletter of the Human Resource Association of the Northeast. Holyoke, MA, pp. 9-10.

Stoneking, B.C., Greenfield, T., Sundby, E.B., and Boltz, S. (1991). Adding trained consumers to case management teams as service coordinators: Program development, research design, and early outcomes. Paper presented at the 119th Annual Meeting of the American Public Health Association, Atlanta, Georgia, November 10-14. 